芒

Documents Collectipf-1 JAN 141963

\section{LOVELACE FOUNDATION} for Medical Education and Research

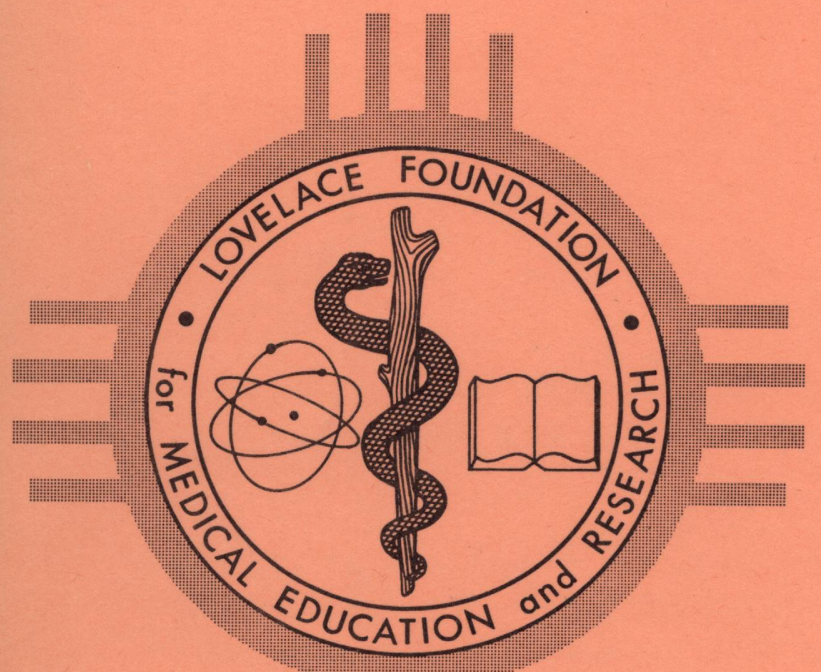

Albuquerque, New Mexico
AEC RESEARCH AND DEVELOPMENT REPORT

UNCLASSIFIED

\section{ON THE CALIBRATION OF CASCADE IMPACTORS \\ by}

T. T. MERCER

September, 1962

\section{ATOMIC ENERGY COMMISSION- LOVELACE FOUNDATION} FISSION PRODUCT INHALATION PROJECT 
LEGAL NOTICE

This report was prepared as an account of Government sponsored work. Neither the United States, nor the Commission, nor any person acting on behalf of the Commission:

A. Makes any warranty or representation, express, or implied, with respect to the accuracy, completeness, or usefulness of the information contained in this report, or that the use of any information, apparatus, method, or process disclosed in this report may not infringe privately owned rights; or

B. Assumes any liabilities with respect to the use of, or for damages resulting from the use of any information, apparatus, method, or process disclosed in this report.

As used in the above, "person acting on behalf of the Commission" includes any employee or contractor of the Commission to the extent that such employee or contractor prepares, handles or distributes, or provides access to, any information pursuant to his employment or contract with the Commission. 
ON THE CALIBRATION OF CASCADE IMPACTORS

by

T. T. Mercer

Submitted as a

Technical Progress Report

to

The Division of Biology and Medicine

United States Atomic Energy Commission

on

Contract No. AT(29-2)-1013

August 31,1962

From the Section of Aerosol Physics

Lovelace Foundation for Medical Education and Research

Albuquerque, New Mexico

U N C LASS I F I E D 


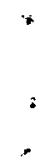

!

? 


\section{PREFACE}

This work is an aspect of investigations concerned with the biological effects of inhaling fission products. It is supported by the Division of Biology and Medicine of the U. S. Atomic Energy Commis sion. The initial contract was awarded June 1, 1960 and has been extended through September 30, 1962. 
$\checkmark$

.

. 


\begin{abstract}
In the following paper, the two customary methods of calibrating cascade impactors are compared. It is shown that the method of determining collection efficiency as a function of particle size for each stage provides information for interpreting results in terms of impactor theory, making it possible to establish adequate jet performance. Two criteria are suggested for expressing impactor performance; the root inertial parameter, $\digamma$, for which collection efficiency equals 0.5 and the slope of the straight portion of the efficiency curve. It is also shown that calibration in terms of the mass median diameter can lead to serious misinterpretation of results.

The method of determining the collection efficiency for each stage is shown to be generally preferable for the interpretation of data obtained from sampling unknown aerosols, but both methods have shortcomings which suggest the need for a more refined method of interpretation.
\end{abstract}


$\checkmark$

t.

$\mathfrak{v}$

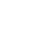




\section{ACKNOW LEDGMENTS}

The author is indebted to Dr。Paul E. Morrow and Dr. Clayton S. White for their advice with respect to the preparation of the manuscript。 
:

$\Sigma$ 
Page

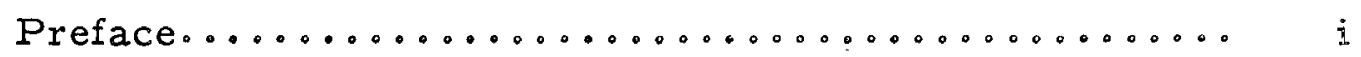

Abstract............................. ii

Acknowledgments ..................... iii

Table of Contents .................... iv

List of Tables ....................... iv

List of Figures $\ldots \ldots \ldots \ldots \ldots \ldots \ldots \ldots \ldots \ldots \ldots \ldots$ v

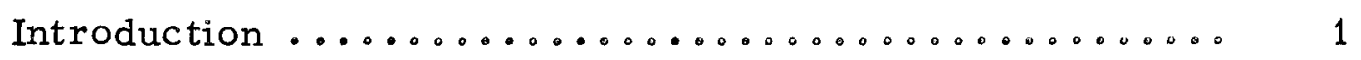

Theoretical Aspects of the Impaction Process ....... 4

Evaluation of Impactor Performance ........... 11

The Inte rpretation of Sampling Data $\ldots \ldots \ldots \ldots \ldots$

a. The Ideal Jet System ............. 20

b. Real Jet Systems .............. 21

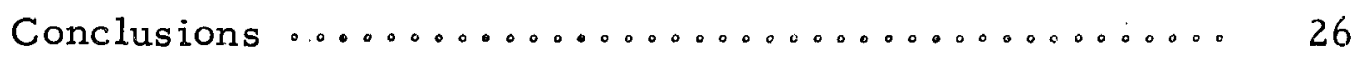

Appendix I. Collection Efficiencies of the Laskin

Impactor................ 27

Appendix II. Collection Efficiencies for the Lippman

Calibration .................... 30

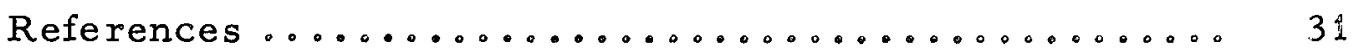

\section{LIST OF TABLES}

Table 1. Characteristics of the Casella Impactor...... 13

Table 2. Characteristics of the Laskin Impactor...... 13 


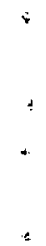

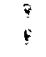

-

= 


\section{LIST OF FIGURES}

Figure

Page

1. Impingement of an Air Jet on a Plane Surface ... 5

2. Theoretical Collective Effichencies as Functions

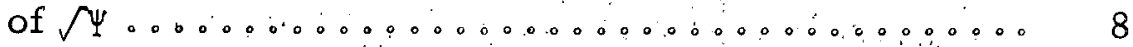

3. The Effect of the Parameter S/W on Collection

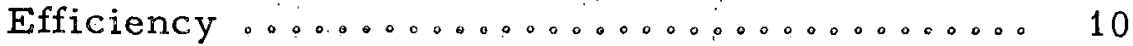

4. Collection Efficiencies of the Casella (a) and the Laskin (b) Impactors................ 14

5. Collection Efficiencies of the First (a) and Second (b) Stages of Several Impactors..... o......

6. Distribution of Particles Among the Various Stages of an Impactor Having. Jets with Efficiency Curves Similar to that of Ranz

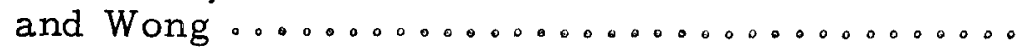

7. Comparison of the Two Calibration Methods

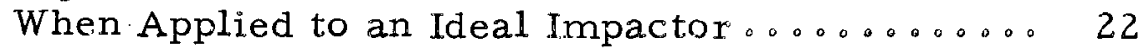

8. Comparison of the Two Calibration Methods When Applied to a Real Impactor ... o. o. 24

9. Efficiencies Curves for the Laskin Impactor .... 28 


\section{ON THE CALIBRATION OF \\ CASCADE IMPACTORS\%}

by

$T . T . M E R C E R$

\section{INTRODUCTION}

The mass distribution of an aerosol is of particular interest both to the industrial hygienist and to the research worker concerned with problems of inhalation toxicology. In practice, the determination of the particle size distribution of an aerosol is most commonly accomplished by collecting a sample suitable for observation in an optical or electron microscope and making direct measurements of particle diameters. This yields a number distribution, showing the relative number of par w ticles within a given increment of size. To make an acceptable estimate of the mass distribution from the number distribution requires a valid mathematical expression for the latter, and even then the estimate is subject to considerable error ${ }_{\imath}$ especially when the range of particle sizes is great. For this reason, it is desirable to have an instrument which provides a direct determination of the mass distribution. At the present time, the only instruments available to do this operate on the principle of cascade impaction first employed by May (1945)。

May's impactor consisted of four impingement stages in series. In each stage, air flowing through a rectangular jet impinged upon a

* This paper will appear in the Arnals of Occupational Hygiene, Vol.6, No. 1 . 
glass slide and was deflected through $90^{\circ}$. Particles of sufficient inertia were unable to negotiate the turn and were collected on the glass slide. By making the jets progressively smaller, causing a reciprocai increase in the velocity through the jet, it was possible to collect progressively smaller particles. Although May was interested primarily in obtaining samples for microscope analysis in which large particles would be fairly represented, he recognized the usefulness of the instrument for deter. mining mass distributions. For all but the first stage he gave "effective drop sizes" (EDS) against which the fraction of the total mass penetrating a given stage could be plotted to obtain an approximate distribution.

The usefulness of the cascade impactor for determining mass dis tributions depends to a large extent on the accuracy of its calibration. The empirical calibration of the EDS values was made as follows: various sprayed mists of unit density drops (dibutyl phthalate) were sampled by the cascade impactor and the samples were counted and sized by two observers. From each count the overall volume distribution curve was computed and plotted and the liquid volume on each stage was also computed. In each case, the cumulative stage-byostage volume starting from stage 4 was plotted on the overall distribution curve. The corresponding drop diameters at these plotted points were taken as the EDS values and the finally presented figures were the averages from all the curves. These EDS values in practice are somewhat larger than the $50 \%$ cutoff values described below。

A calibration must include two steps: first, the pexformance of each stage is assessed by using the impactor to sample an aerosol of known physical characteristics: then, on the basis of this performance, 
"calibration constants" are determined for each stage to provide a means of interpreting data obtained subsequently from samping othe aerosols of interest. Since May's work appeared, a number of cascade impactors have been described in the literature and several calibrations have been reported. Two other methods of calibration have been used. In ore method, the collection efficiency of each stage, that is, the ratio of the number of particles of a given size retained at a given stage to the total number of particles of that size entering that stage, is determined as a function of paricle size for a test aerosol. The particle size for which the collection efficiency of a particulax stage is equal to 0.5 is then taken as an effective. "cutoff" size and represents the callbration constant for that stage. This method, which will be referred to hereafter as the effective cutoff method, was used by Ranz and Wong (1952) and Mitchell and Pilcher $11958 \%$. It has the advantage that the calibration constants are not functions of the particle size distribution of the aerosol used in making the calibration, as are EDS values. Wher other aerosols are sampled, the cumulative mass collected or all stages up to, and including, a given stage is plotted, as percent of total mass collected, against the calibration constant for that stage. The calibration constartis adjusted for differences in sampling flow rate and densty of the aerosol particles. Logarithmic probability paper is used for plotting the expex imental points, under the assumption that the mass is "lograormally" distributed with respect to particle size.

In the other method of calibration, a sample of a krown aerosol is obtained and the mass median diameters for the particles collected at each stage are calculated from the number distribution obtained by 
measurements made with the optical microscope. (For a given stage, half of the mass collected on that stage is associated with particles having diameters larger than the mass median diameter./ These mass median diameters, adjusted for differences in sampling conditions, are then used to determine the mass distribution of other aerosols. For a given stage, half the mass collected on that stage plus all the mass coli lected on previous stages is plotted, 2 s percent of total mass collected, against the mass median diameter for that stage, again using logarithmic probability paper. This method was introduced by Sonkin (1946), who

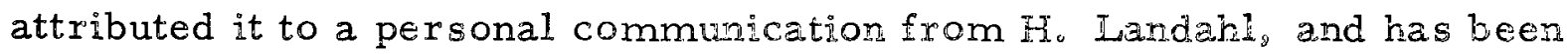
used by Laskin (1949), Heneveld (1959) and Lippmar (1959)。 Hereafter. it will be referred to as the M.M.D. method.

In this discussion, the relative mexits of these tro methods will be compared with respect both to the evaluation of impactor performance and to the application of the results to the interpretation of samping data. It will be necessary first, however, to review briefly the theory of the impaction process in order to establish the physical basis for making the comparison.

Theoretical Aspects of the Impaction Process.

When a jet of air from a rectangular slot impinges normally upon a plate as shown in Fig。 1 , it splits into two equal streams moving in opposite directions:

Because of its relatively laxge inertia, a particle moving with the air along a given stream line deviates from that line as the air de. flects upon approaching the plate. If that deviation is sufficiently large, the particle will strike the plate and, presumably, be retained there. 


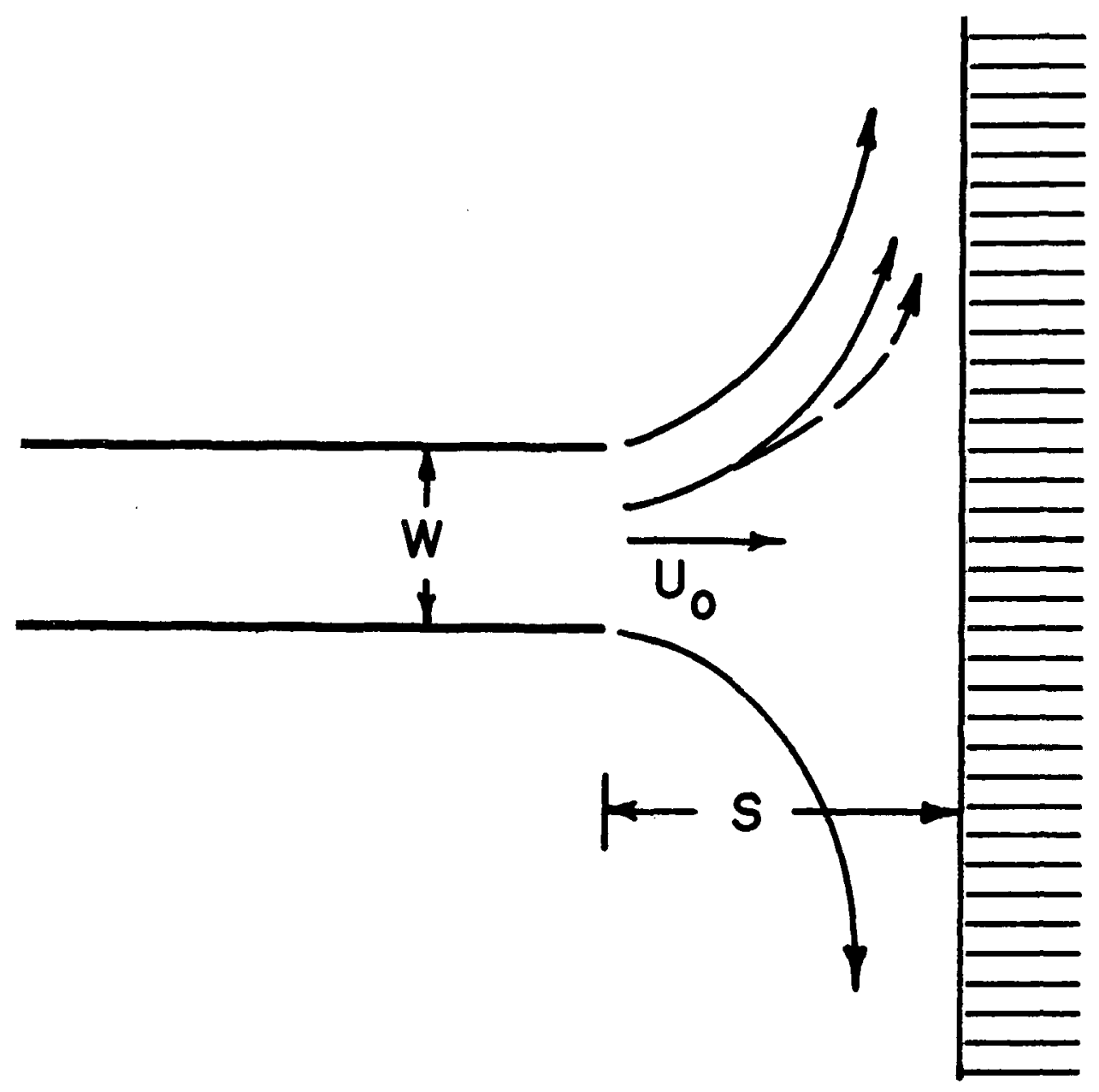

Fig. 1. Impingement of an air jet on a plane surface. 
Davies and Aylward (1951) studied this impaction process theo retically for rectangular jets. They assumed the air was an ideal flaid in order to calculate the flow field, while the resistance of the air to the relative motion of the particle was assumed to be given by Stokes" law. It was further assumed that the particles were spherical and that any particle coming in contact with the plate was permanently retained.

When the equations of motion of the particle were expressed in dimen sionless variables, the physical characteristics of the particle and the jet appeared only in a single dimensionless parameter $P_{2}$ giver by

$$
P=\frac{\rho d^{2} U_{o}}{9 \eta W}
$$

where $p$ is the particle's density, $d$ is its diameter $\eta$ is the viscosity of the air, $W$ is the width of the jet, and $U_{0}$ is the velocity of both air and particle as they leave the jet. Physically, $P$ is the stopping distance of a particle of diameter $d$ entering still air at an initial velocity $\mathrm{U}_{\mathrm{O}^{\circ}}$ meas ured in units of one-half the jet width.

For a given flow field, equal values of $\mathrm{P}$ would indicate equal collection efficiencies, whatever the values of the several physical quantities included in $P$. For a given jet, however, Davies and Aylward found the flow field was markedly affected by the jetwcollector separation, that is, the distance of the jet orifice from the collector plate. They showed that the efficiency of impaction was a function of the two dimensionless parameters, $P$ and $S / W$, the latter being the ratio of the jet-collector separa-

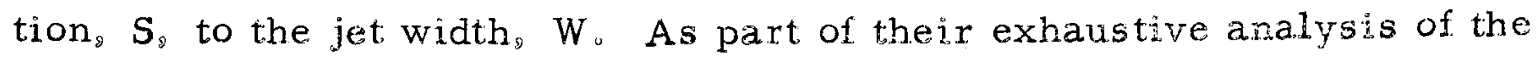
impaction process, they obtained curves of efficiency as a function of $P$ 
for a number of values of $\mathrm{S} / \mathrm{W}$.

Ranz and Wong (1952) studied the impaction frocess theoretically and experimentally for both round and rectangular jets. They did not specify the value of $\mathrm{S} / \mathrm{W}$ for their theoretical calculations: however, the approximations they introduced with respect to boundary conditions and velocity profiles in effect provided them with a value of 0.5 for $S / W$ for rectangular jets. They expressed the equations of motion in terms of a dimensionless parameter, $\Psi$, given by

$$
\Psi=\frac{\rho d^{2} \cdot U_{0} C}{18 \eta W}
$$

where $C$ is a factor which corrects the Stokes ${ }^{8}$ law of viscous resistance for the effect of "slip" as the size of the particle approaches the length of the mean free path of air molecules and the other quantities are as defined above for $P$. They calculated collection efficiencies for various values of $\psi_{2}$ and presented a theoretical curve of efficiency a.s a function of $\sqrt{ }$, since this quantity is directly proportional to the particle diameter, do This curve is shown in Fig。 2 , together with the theoretical curve for $S / W=0.5$ obtained by interpolation of the curves of Davies and Aylward.

Although May did not make a theoretical study of the impaction process, his dimensional analysis should be mentioned because it led to

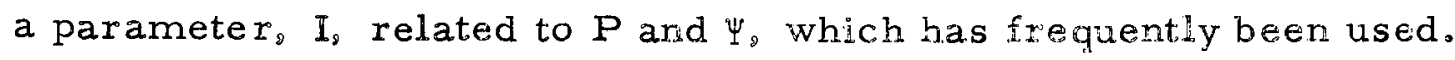
This parameter was given by

$$
I=\frac{\rho d^{2} U_{o}}{\eta W}
$$




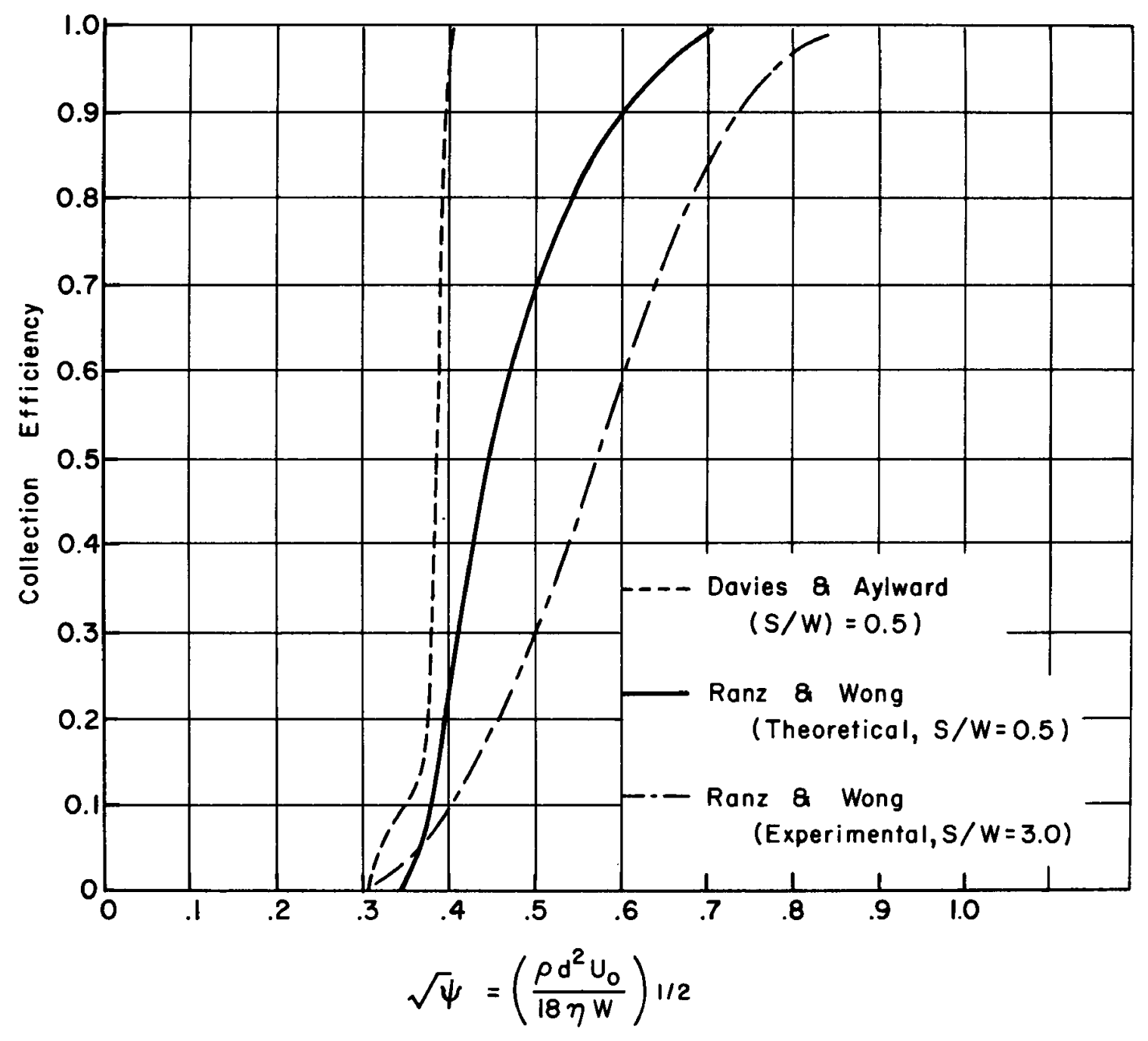

Fig. 2. Theoretical collection efficiencies as functions of $\mathbb{A}$. 
where the symbols have the same meaning as in the definitions of $P$ and \% The relationships among these three parameters are

$$
P=(2 / C) \Psi=(1 / 9) I
$$

There has been very little work done to test the theoretical curves of either Davies and Aylward or Ranz and Wong. The latter did carry out an experimental study of the impaction process: however, they used impactor systems in which S/W was very nearly equal to 3, compared with a value of 0,5 for their theoretical calculations. The curve obtained experimentally, which is also shown in Fig, 2, had the same shape as their theoretical curve but was displaced to higher values of $\sqrt{ } \psi$, the point corresponding to a collection efficiency of 0.5 moving from $\sqrt{\Psi}=$ 0.44 to $\sqrt{ } \psi=0,57$. In the work of Davies and Aylward the collection

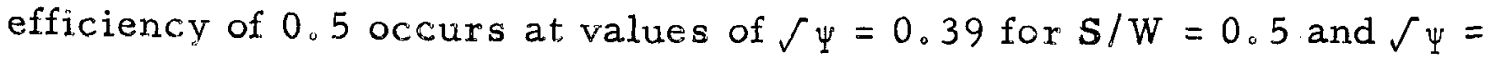
0.56 for $S / W=3$ 。

The significance of the ratio $\mathrm{S} / \mathrm{W}$ can be shown best by plotting $\sqrt{\Psi}$ corresponding to a collection efficiency of 0.5 against $S / W$. Again, the results of Davies and Aylward were used to obtain the curve shown in Fig。 3。 As S/W increases beyond 1, the change in collection efficiency is quite slow, but at small values of $S / W$, small changes in $S$ may bring about relatively large changes in efficiency. Thus an impactor designed for optimum efficiency must be designed also to accurately maintain the distance S。

In making comparisons between jets, it must be kept in mind that

* Because the parameter $\sqrt{ } \Psi$ appears to have been used more frequently than $\sqrt{P}$, it is used here, even though this discussion is based largely on the theoretical results of Davies and Aylward. 


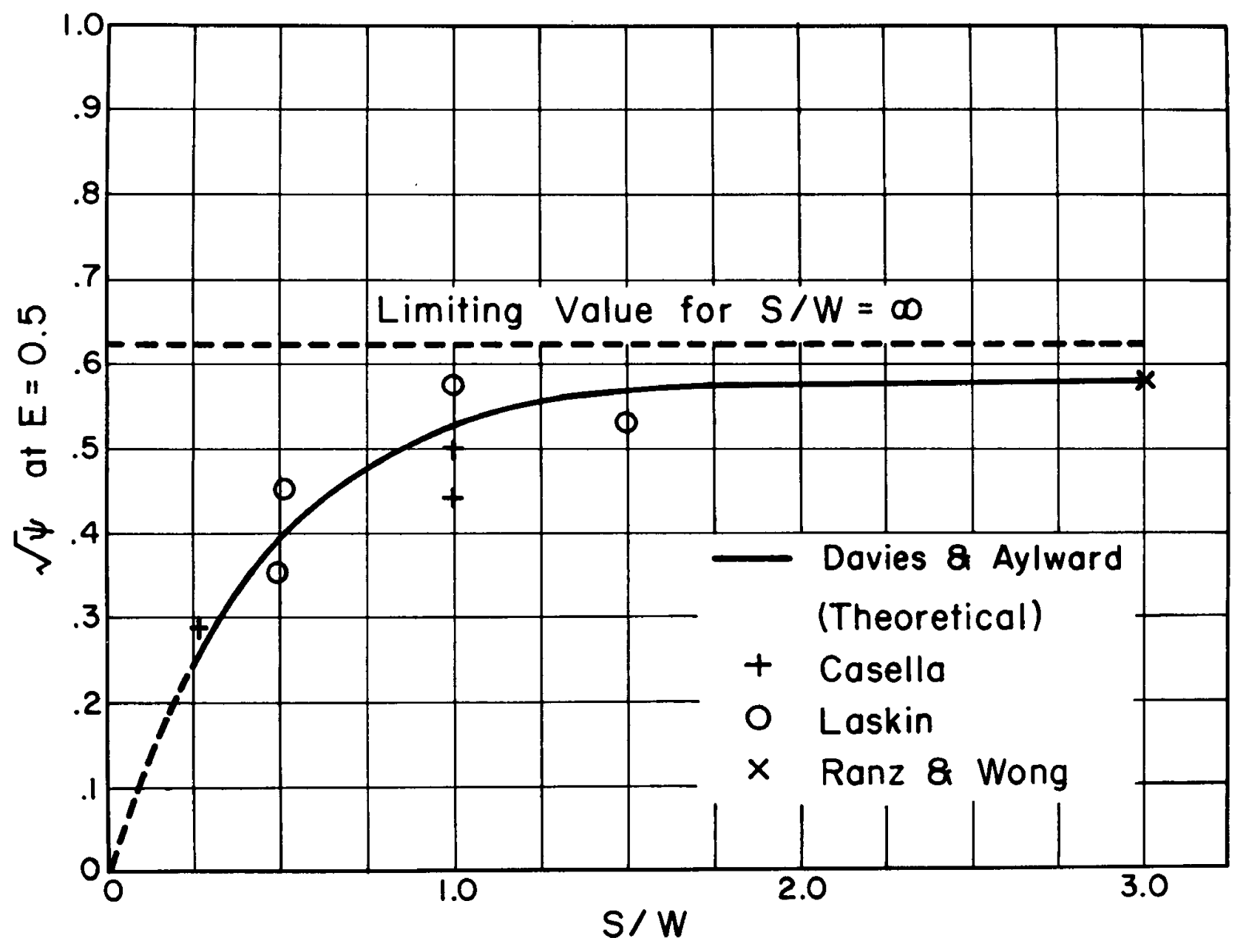

Fig. 3. The effect of the parameter $\mathrm{S} / \mathrm{W}$ on collection efficiency. 
the parameters $\Psi$ and $\mathrm{P}$ were derived for an impaction system in which the air stream is split symmetrically and diverted in opposite directions. When this is not the case, as when the full steam is deflected in one direction, the formulas for $P$ and $w$ are not strictly applicable. However, they can be adjusted to approximate the case of unidirectional deflection by replacing the slit width, $W$, with $2 \mathrm{~W}$ 。

Evaluation of Impactor Performance.

It is apparent that the curves of efficiency versus particle size, obtained for the various stages when the effective cutoff method is used in calibrating the cascade impactor, can be converted readily to curves of efficiency versus $\sqrt{\Psi}$ for comparison with the theoretical curves. While there has not been sufficient experimental support of the theoretical curves to provide definitive criteria for the adequacy of jet performance, the calibration data for the Casella* version of the May impactor and for the Laskin impactor make it possible to establish a minimum acceptable performance.

The calibrations of these two impactors, which were similar but

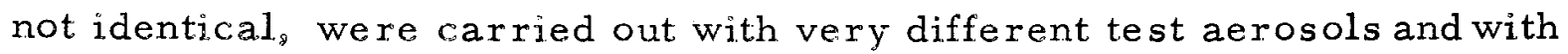
different methods. The Casella impactor was calibrated with liquid droplets of unit density, and the necessary data for applying the effective cutoff method are supplied with the instrument. Laskin calibrated his impactor on a $\mathrm{UO}_{2}$ dust, the particles of which were irregular in shape but roughly spherical and had a density of $10.9 \mathrm{gm} / \mathrm{cm}^{3}$. He used

*The Casella impactor is a commercially available version of the May impactor. It is described in reference 2, from which the data referring to it were obtained. 
the $M \cdot M \cdot D$. method in his calibration. The pertinent data concerning their impactors and sampling conditions are shown in Tables 1 and 2. For the first and second stage jets, in which the air stream is deflected primarily in one direction, $S$ is taken as the average distance of the collector from the jet, and the effective value of $S / W$ is equal to the actual value of $S / 2 W$ 。

The Casella calibration could be converted to the appropriate $\sqrt{ } \Psi$ scale without difficulty。 The results are shown in Fig。4a. Since Laskin used the $M . M \circ D$. method, however, the conversion of his data to the curves shown in Fig。 $4 \mathrm{~b}$ was more involved and is described in detail in Appendix I.

Two criteria that appear suitable for evaluating jet performance from curves of efficiency versus $\sqrt{\Psi}$ are the slope of the straight portion of the curve and the position of the curve with respect to the $\sqrt{ } \Psi$-axis. The latter criterion can be defined in terms of the value of $\sqrt{\Psi}$ at which the collection efficiency equals 0.5 . These values for the curves of Casella, Laskin, and Ranz and Wong are shown in relation to the theoretical curve of Davies and Aylward in Fig。3. The slopes of the curves of Casella and Laskin vary between about 2.2 and 5.0 , while the slope for the Ranz and Wong curve is 2.7 .

Both of these criteria are functions of the parameter $\mathbf{S} / \mathrm{W}$, the slope decreasing and the $\sqrt{\Psi}$ increasing with increased values of $\mathrm{S} / \mathrm{W}$. On the basis of the information presented above, the curves of efficiency versus $\sqrt{\Psi}$ should have straight segments for which the slope is not less than 2.7 and the value of $\sqrt{\Psi}$ at which the collection efficiency is 0.5 should not exceed 0.61 . Even the latter value should be viewed 


\begin{tabular}{|c|c|c|c|c|}
\hline Stage & $\begin{array}{r}\text { Jet } \\
\text { Width; W } \\
\end{array}$ & $\begin{array}{l}\text { Jet-Collector } \\
\text { Separation, S }\end{array}$ & $\begin{array}{c}\begin{array}{c}\text { Jet Velocity } \\
\mathrm{U}_{\mathrm{o}}\end{array} \\
\end{array}$ & $\begin{array}{c}\text { Effective } \\
\mathrm{S} / \mathrm{W} \\
\end{array}$ \\
\hline 1 & $0.65 \mathrm{~cm}$ & $0.35 \mathrm{~cm}$ & $2.37 \times 10^{2} \mathrm{~cm} / \mathrm{sec}$ & 0.27 \\
\hline 2 & 0.2 & $\infty$ & $1.02 \times 10^{3}$ & $\infty$ \\
\hline 3 & 0.075 & 0.075 & $2.75 \times 10^{3}$ & 1.0 \\
\hline 4 & 0.027 & 0.027 & $7.7 \times 10^{3}$ & 1.0 \\
\hline
\end{tabular}

TABLE 1. CHARACTERISTICS OF THE CASELLA IMPACTOR

$\begin{array}{ccccc}\text { Stage } & \begin{array}{c}\text { Jet } \\ \text { Width, W }\end{array} & \begin{array}{c}\text { Jet-Collector } \\ \text { Separation, }\end{array} & \begin{array}{c}\text { Jet Velocity } \\ \text { U }\end{array} & \begin{array}{c}\text { Effective } \\ \mathrm{S} / \mathrm{W}\end{array} \\ 2 & 0.44 \mathrm{~cm} & 0.41 \mathrm{~cm} & 3.78 \times 10^{2} \mathrm{~cm} / \mathrm{sec} & 0.47 \\ 3 & 0.13 & 0.135 & 1.28 \times 10^{3} & 0.52 \\ 4 & 0.10 & 0.150 & 1.66 \times 10^{3} & 1.50 \\ & 0.06 & 0.061 & 2.77 \times 10^{3} & 1.02\end{array}$

TABLE 2。 CHARACTERISTICS OF THE LASKIN IMPACTOR 
with suspicion when the value of $S / W$ is known to be small.

Among the reasons for deviation of the experimental curves from the theoretical curves are disaggregation of agglomerates particle shapes that are markedly non spherical, rebound of particles upon strik ing the collection plate or particles previousiy deposited, ard re-entrain. ment of captured particles. The first two difficulties can be avoided by careful choice of a test aerosol, while the last two can be minimized by the use of adhesive coatings on the collecting plates and by avoiding heavy deposits at any stage。 Rebound and rewentrainment reduce the slopes of efficiency curves and displace them to kigher values of $\sqrt{\Psi}$. It is apparent that a calibration carried out according to the effective cutoff method can provide an insight into the extent of these difficulties.

On the other hand, the M。M.D. method provides no basis at all for a logical evaluation of jet periormance. Calibrations of this type can lead, in fact, to serious misinterpretations of results, when com* parisons are made among different impactors. The dargers inherent in this method are strikingly demonstrated by the work of Lippman (1959) who used it in recalibrating a. standard Casella impactor. He sampled $\mathrm{U}_{3} \mathrm{O}_{8}$ aerosol and treated the data according to the effective cutoff method using the calibration constants of May and of Ranz and Wong and according to the $M \circ M_{\circ} D$ 。 method using the constants of Laskin。All calibration constants were adjusted for differences in sampling flow rate. There was a very marked disagreement among the mass distributions obtained in this way and an even more marked disagreement between them and the mass distributions obtained with his own calibration constants. He concluded that this comparison "created doubts about the 
accuracy of all previously published calibration data and the applicability of cascade impactors for particle sizing."

In making his comparison, Iuppman assumed that in each impactor the particle mass would be distributed among the different stages exactly as he observed it in his Casella instrument. For this to be the case, it would be necessary for corresponding stages of the different impactors to have the same collection efficiencies for similar particles under similar sampling conditions. To test this, curves of collection efficiency as a function of $\sqrt{ } \rho d$, for $\rho$ in $g r a m s / \mathrm{cm}^{3}$ and $d$ in microns. were calculated for the first two stages of each of the impactors of interest. These are shown in Fig. 50 The curves for Laskin 's impactor were obtained from those shown ir Fig. 9 for collection with dry slides. They have been corrected to a sampling flow rate of 17.5 liters per minute. Had they been corrected to the case of collection with resin coated slides, they would be displaced to even lower values of $\sqrt{ }$ pd: how ever the curves for "dry collection" serve to make the desired pointo

The curves for Ranz and Wong were calculated from their exper imental curve of Fig。2, assuming the ratios of the linear velocity through the jet to jet width were 314 per second and 5,100 per second for the first and second stages respertively, as they are for the Casella impactor。

The curves for the Lippman calibration were calculated in the manner indicated in Appendix. II, while the curves for the May impactor were taken from reference 6 。

It is obvious from these curves that the distribution of mass in a Laskin impactor, or in one made up of jets of the type used by Ranz and 

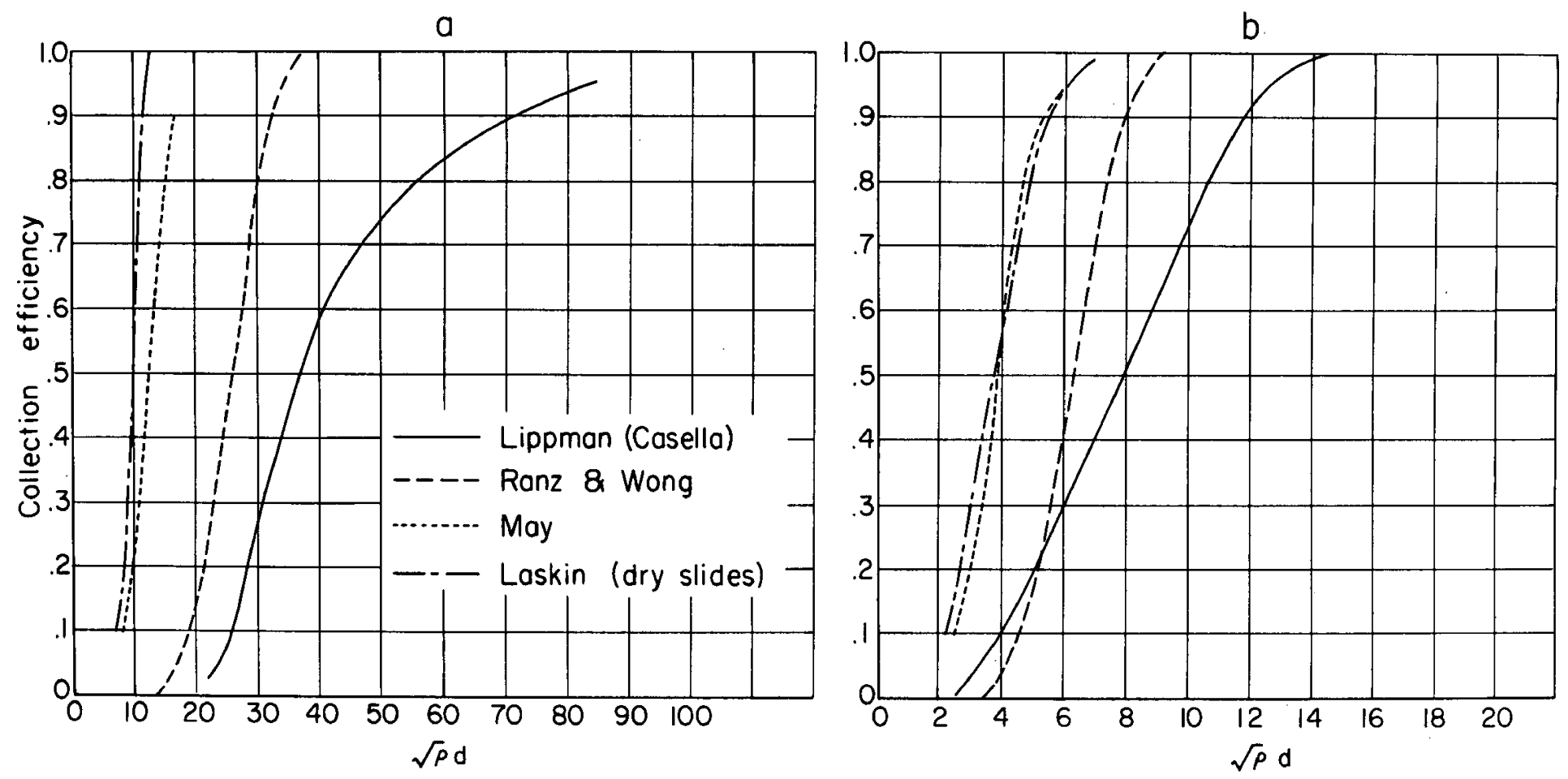

Fig. 5. Collection efficiencies of the first (a) and second (b) stages of several impactors. 
Worg would differ greatly from that observed by Lipprnan. Thus Lippman's comparison and the conclusions drayn from it are quite in valid. Difficulties such as this illustrate one of the serious stortcomings of the method of calibrating impactors in terms of mass median diameters and emphasizes the need for reporting impactor performance in a consistent and logical manner such as that offered by the effective cutoff method。

The Interpretation of Sampling Data.

The efficiency curves presented above indicate the fate of individ ual particles or entering a given stage. The mass fraction collected at that stage, however, is a function both of the collection efficiency of the jet and the mass distribution of the aerosol being sampled. An example of the results that could be expected with a cascade impar:or of four jets, each having an efficiency curve similar to the experimental ctirve of Ranz and Wong is shown in Fig。6。 It was assumed that the jets and flow rate were chosen so that the diameters of unit density particles for which the collection efficiency was equal to 0.5 were $16 \mu, 8 \mu, 4 \mu$ and $2 \mu$ respec:tively for the four stages. The aerosol was assumed to be made up of unit density particles having a mass distribution for which the median

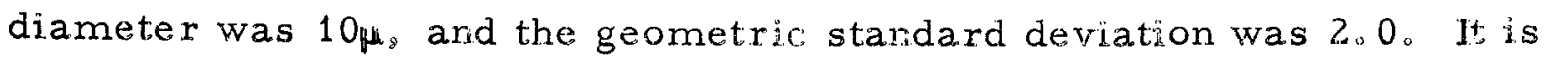
apparent from the spread of particles on each stage that the determina. tion of the mass distribution from the gross measurements of mass colw lected on the individual stages is not a simple matter.

The estimation of the mass distribution as made with the effective cutoff method is based on the assumption that the efficiency curves can be approximated by step functions, with the step for a given stage 


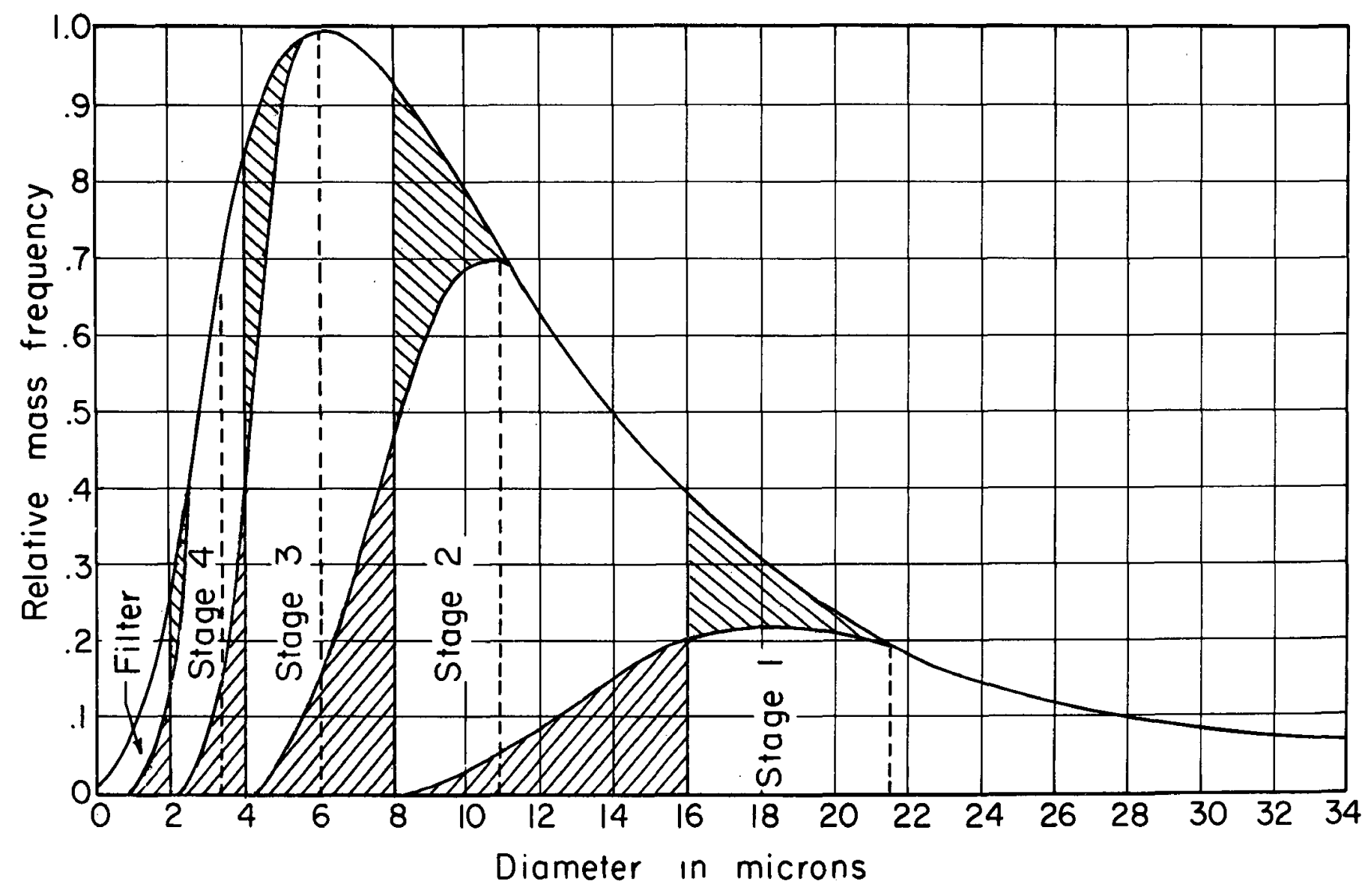

Fig. 6. Distribution of particles among the various stages of an impactor having jets with efficiency curves similar to that of Ranz and Wong. 
occurring at the calibration constant for that stage. The source of exror in this method is demonstrated in Fig。 6 , where the vertical dashed lines represent the calibration constants of the different stages. For each stage, the upper shaded area measures the amount assumed to be col lected when actually it is not and the lower area measures the amount assumed to escape when actually it is collected. The accuracy of the effective cutoff method depends on how nearly equal these two areas are.

The mass median diameters are also indicated in Fig。6。 Intui tively, one would expect that for a particular impactor these diameters would vary considerably with the particle size distribution of the aerosol used incalibration. That this is so, can be seen by replacing the aerosol assumed in Fig. 6 with one having a different mass distribution.

A really satisfactory comparison of these two methods of interpreting sampling data would require ar analysis of the results to be expected from a great many different particle size distributions. A few ex amples are instructive, however, and suggest what might be expected generally. In the following discussion, the relative merits of the two methods are considered first from the standpoint of an ideal jet system and then from the standpoint of a practical system. The Ideal Jet System。

For an ideal jet, the curve of efficiency versus $\sqrt{ } \Psi$ would be a step function, with the step occurring at some critical value, $\sqrt{ } \Psi C_{9}$ such that for all values of $\sqrt{ } \Psi<\sqrt{\Psi}$ c the collection efficiency would be zero and for all other values it would be one. To compare the two methods of calibration for this ideal system, assume that an impactor is made up of four of these jets in series followed by a perfect filter. The flow rate and jet sizes are chosen so that the cutoff sizes for particles of unit 
density, are $16 \mu, 8 \mu, 4 \mu$ and 2 a respectively for the four stages。 Assume this impactor is calibrated by collecting a sample of an aerosol of particles of unit density for which the mass median diameter is known to be exactly 5.0 0 and the geometric standard deviation is exactly 2.0 . The mass fractions collected in the various stages are determined and compared with the known curve to establish their cutoff dimensions and mass median diameters. Obviously, the exact cutoff dimensions will be obtained. The mass median diameters will be found to be $19.8 \mu, 10.3 \mu$, $5.6 \mu$, and $3.0 \mu k$.

Having calibrated the ideal jets, the impactor may be used to sample other aerosols. Whatever the distribution of these aerosols, the effective cutoff method will always yield the true parameters of the distribution。 This is not true of the M.M.D. method, as is apparent in Fig。 7. If used to sample two aerosols of the same geometric standard deviation as the test aerosol but having mass median diameters of $2.0 \mu$ arid 15. Op respectively, this method would yield the points shown for each line. In fact, for similar geometric standard deviations the method will tend to move the mass median diameter toward that for which it was calibrated. For similar mass median diameters the method will tend to rotate the line to provide a geometric standard deviation nearer to that of the calibration aerosol. For an ideal impactor, the effective cutoff method would unquestionably be the method of choice.

\section{Real Jet Systems.}

Since ideal impactors are not available, it is necessary to com w pare the two calibration methods on the basis of efficiency curves that can actually be obtained. To do this, the impactor assumed previously 


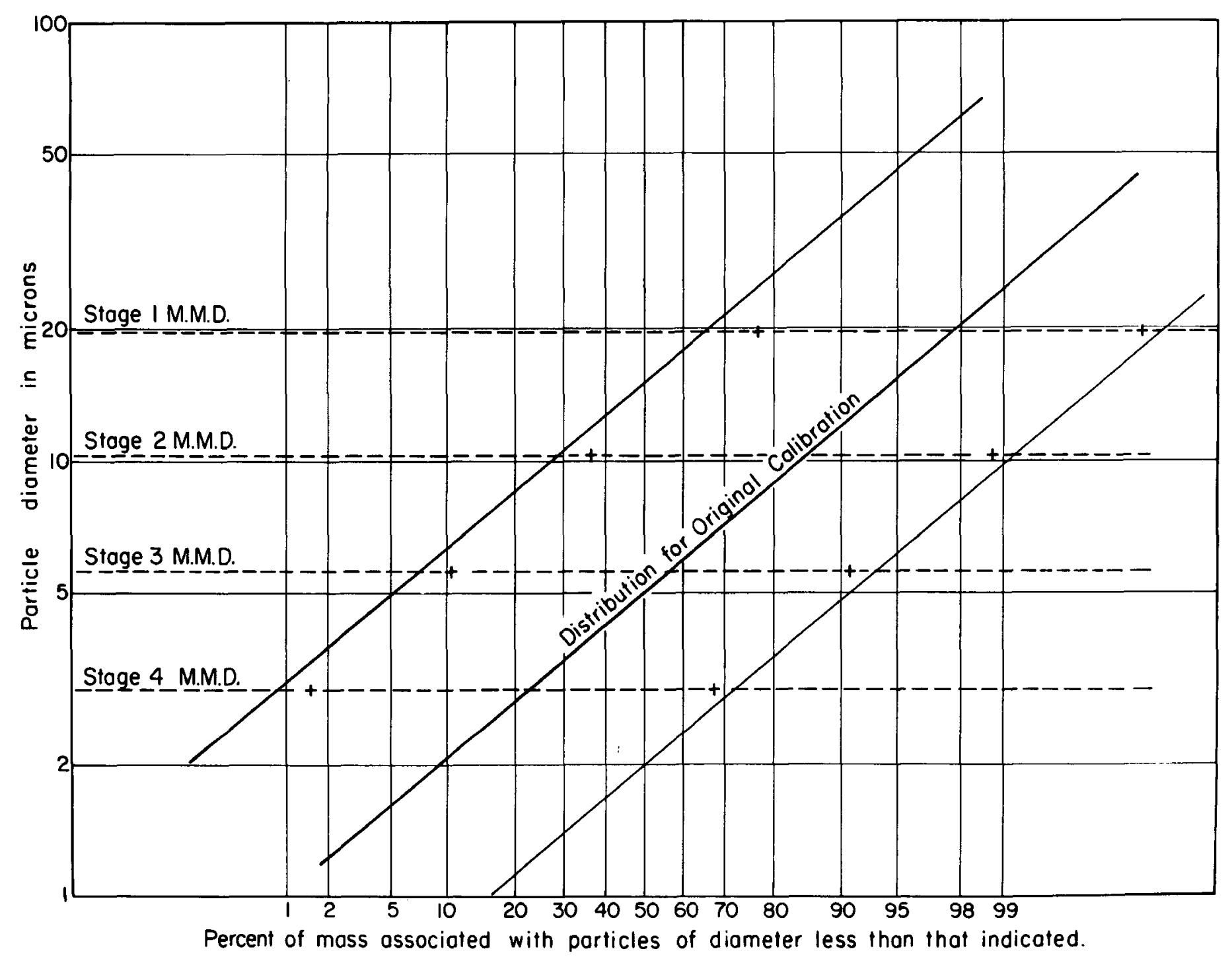

Fig. 7. Comparison of the two calibration methods when applied to an ideal impactor. 
for Fig. 6 is used。 If such an impactor were calibrated by sampling a known aerosol of mass median diameter equal to $10.0 \mu$ and geometric standard deviation equal to 2.0 , the calibration would yield cutoff diameters of $16.0 \mu_{2}, 8,0 \mu, 4.0 \mu$, and $2.0 \mu$ for the four stages. It would yield mass median diameters of $21,5 \mu, 10,0 \mu, 6,0 \mu_{8}$ and $3.35 \mu$. If these calibration constants are applied to the results obtained when the impactor is used to sample aerosols having the same geometric standard deviations as the calibration aerosol, but different mass median diameters, it is found that both methods yield distributions displaced toward the original calibration. If aerosols having the same mass median diameters as the test aerosol but different geometric standard deviations are sampled, it is found that both methods yield comparable results if the first stage is ignored in the $M \circ M \circ D$ 。 method, If it is not, this method tends to rotate the distribution to a slope more nearly like that of the distribution of the calibration aerosol. Examples of both cases are shown in Fig。 8 。

While this comparison of calibration methods has been based on hypothetical samples, there is no reason to believe that the results would be different in practice. If the basic assumption that the efficiency curves are reproducible is not valid, then no calibration method will be successful. On the basis of these hypothetical samples, then, one would conclude that the effective cutoff method is generally preferable to the $M \circ M \circ D$. method。 It will give results that are comparable to, or better than, those of the latter, without requiring that the data from the first stage be discarded. Furthermore, as the jet efficiency improves, the effective cutoff method becomes increasingly accurate. It is apparent, 


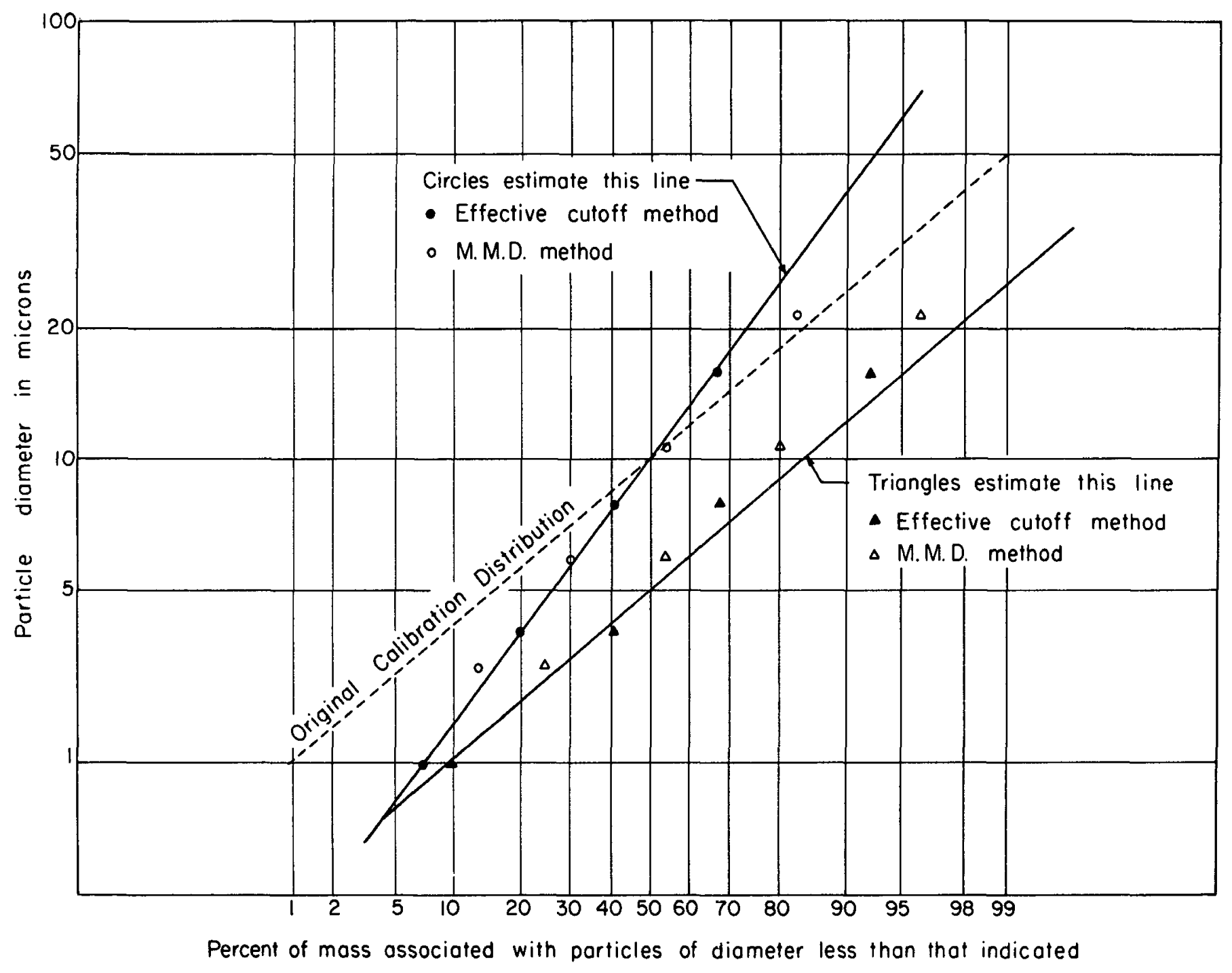

Fig. 8. Comparison of the two calibration methods when applied to a real impactor. 
however, that for the interpretation of sampling data both methods leave something to be desired. It would seem that the use of the siraight line approximation (see Appendix I) to the efficiency curve, which is quite good even for the Ranz and Wong curve, might be fruitfully explored as a means of making an analytical determination of the mass distribution. 


\section{CONCLUSIONS}

Cascade impactors should be calibrated by determining collection efficiency as a function of particle size for each stage. The curves obtained should be converted to collertion efficiency as a furction of $\sqrt{ } \Psi$ $=\left(\rho \mathrm{d}^{2} \mathrm{U}_{\mathrm{C}} \mathrm{C} / 18 \eta \mathrm{W}\right)^{1 / 2}$, the root inertial parameter, for comparison with theoretical curves.

Jet performance can be evaluated in terms of two criteria, the slope of the curve of efficiency versus $\sqrt{ } \Psi$ and the value of $\sqrt{ } \Psi$ for which the collection efficiency is 0,5 . The latter should not be more than 0,61 and the slope should not be less than 2.?.

The method of calibrating impactors by determining the mass median diameters of the various stages for a test aerosol does not provide information concerning the proper functioning of the jets and can lead to serious misinterpretation of impactor performance.

The use of effective cutoff diameters in the interpretation of data obtained from sampling unknown aerosols is preferably to the use of mass median diameters: however, neither method is entirely adequate and optimum use of the cascade impactor for determining mass distributions will require the development of more refined methods of data interpretation。 


\section{APPENDIX I}

\section{Collection Efficiencies of the Laskin Impactor.}

In order to obtain optimum conditions with respect to microscopy, Laskin found it necessary to use dry collection slides and coat the samples with selenium. His data (reference 5, page 493 ) have been used to calculate the curves for stages 1 and 2 shown in Fig. 9 and labelled "dry collected" Under normal sampling conditions, however, the collecting slides were resin coated and collection efficiencies were considerably better than these curves indicate. While data comparable to those used in calculating the "dry collected" curves are not available to calculate the efficiency curves for the various stages when the slides are resin coated, they can be estimated in the following way. When sampling a $\mathrm{UO}_{2}$ dust having an overall mass median diameter of $1.53 \mathrm{i}$ and a geometric standard deviation of 2.23 , Laskin obtained mass fractions of 0.232 , $0.461,0.164$, and 0.087 respectively for the four jet stages, and 0.056 for the fifth stage filter. It was assumed thatcurves of efficiency versus particle size could be approximated by a straight line. Using a method reported by Mercer et.ab.(1960), it was possible to determine efficiency curves for the four stages which would lead to the desired mass fractions. These are shown in Fig. 9 as "approximations, resin collected"。 Estimated efficiency curves for stages 1 and 2 for collection with resin coated slides were obtained by displacing the "dry collected" curves until they were superimposed on the corresponding straight line approximations. These estimated efficiency curves appear quite plausible. Thexe are no data against which to check the straight line approximations 


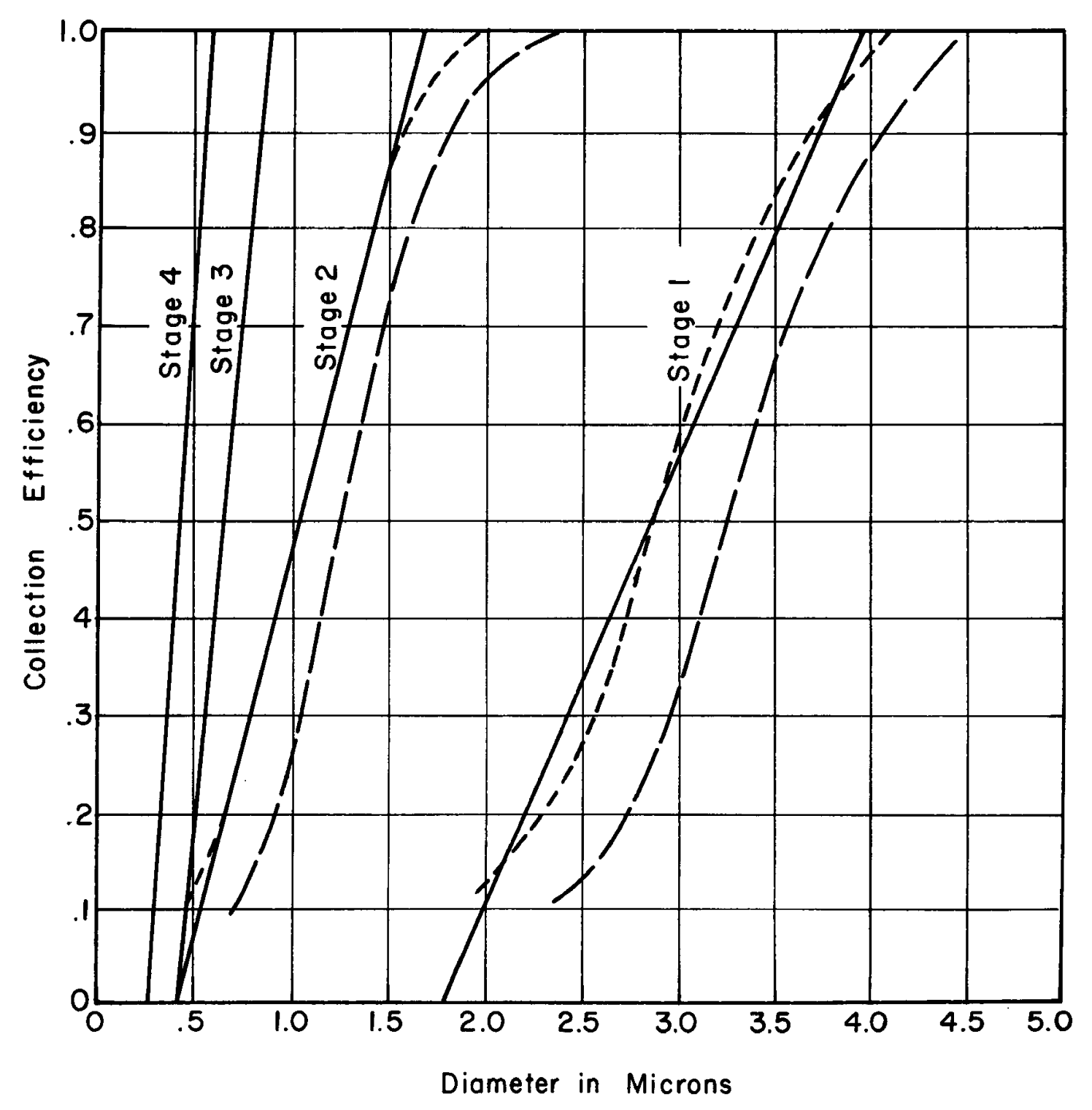

Fig. 9. Efficiencies curves for the Laskin impactor 
for stages 3 and 4; however, the closeness of the stage 2 empirical curve to a straight line supports the assumption that this approximation is reasonable. The estimated efficiency curves for stages 1 and 2 and the straight line approximations for stages 3 and 4 have been used to calculate the curves of efficiency as a function of $\sqrt{ } \Psi$ which are shown in Fig。 $3 \mathrm{~b}$. 


\section{APPENDIX II}

Collection Efficiencies for the Lippman Calibration.

The collection efficiency of the $i$ th impactor stage for particles of diameter $d$ is given by

$$
E_{i}(d)=\frac{m_{i}(d)}{m_{0}(d)=\sum_{j=1}^{i=1} m_{j}(d)}
$$

where $m_{j}(d)$ is the mass on the $j=$ stage associated with particles in a differential element about diameter $d_{2}$ and $m_{0}(d)$ is the mass of the original aerosol in the same size increment. Lippman found the particles to be "log=normally" distributed on each stage, so that

$$
m_{j}(d)=\frac{M_{j}}{\sqrt{2 \pi 1 n \sigma_{j}}} \exp \left[-\left(1 n d-1 n d_{j}\right)^{2} / 21 n^{2} \sigma_{j}\right](1 n d)
$$

where $M_{j}$ is the total mass found on the $j$ th stage and $d_{j}$ and $\sigma_{j}$ are the mass median diameter and geometric standard deviation respectively of the distribution on the $j-$ th stage。 


\section{REFERENCES}

Cascade Impactor Instruction Leaflet, 3018/RD,C.F。Casella and Co。 Ltd. , London。

Davies, C.N. and Aylward, M。 The trajectories of heavy, solid partio cles in a two-dimensional jet of ideal fluid impinging normally upor a plate。 Proc。.Phys: Soc。B, LXIV, 889-911 (1951)。

Heneveld, W.H. Fifth Occupational Health Conference, University of Texas, Austin, Texas。 Andersen Sampler Calibration (1959).

Laskin, S. Pharmacology and Toxicology of Urarium Compounds. Ed. by Voegtlin, Co and Hodge, H.C. Vol。 I, ppo463-505, McGraw Hill Book Co., New York City. (1949)。

Lippman, Mo Review of cascade impactors for particle size analysis and a new calibration for the Casella cascade impactor。A.I.H.A.J。 $20: 5,406-416(1959)$.

May, K.R。 The cascade impactor: an:instrument for sampling coarse aerosols。 JoSci。Inst。 $22,187-195$ (1945)。

Mercer, $T \circ T$, Mills, $C_{0} \cdot M_{0}$ and Gibb, $A . R$. Collection efficiencies of a cascade filtration device employing wire gauzes. Ann。Occ. Hyg. 1. $301-313(1960)$ 。

Mitchell, R.I. and Pilcher, JoM。 Design and calibration of an improved cascade impactor for size analysis of aerosols。 Proc。Fifth $A_{0} E_{0} C_{0}$ Air Cleaning Conf。 TID-7551, pp, 67 84, OTS, Depto of Commerce, Washington, D。C 。(1958)。

Ranz, W.E。 and Wong, J.B. Impaction of dust and smoke particles on surface and body collectors. Ind. Eng。Chem。44, $1371=1381$ (1952)。

Sonkin, L.S. A modified cascade impactor. A device for sampling and. sizing aerosols of particles below one micron in diameter. J.Ind. Hyg。 and Toxicol。28, 269 (1946)。 


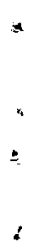

.

. 
1

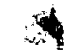

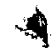

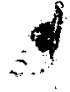




\section{.}

、 